Alba Janeth Ortiz-Andrade; Darwin Gabriel García-Herrera; Carlos Marcelo Ávila-Mediavilla; Juan Carlos Erazo-Álvarez

\title{
http://dx.doi.org/10.35381/e.k.v3i1.1017
}

\section{Influencia del nivel instruccional familiar en el proceso de enseñanza aprendizaje en Educación General Básica}

\section{Influence of the family instructional level in the teaching-learning process in} Basic General Education

\author{
Alba Janeth Ortiz-Andrade \\ alba.ortiz.13@est.ucacue.edu.ec \\ Universidad Católica de Cuenca, Azogues \\ Ecuador \\ https://orcid.org/0000-0002-4472-8664 \\ Darwin Gabriel García-Herrera \\ dggarciah@ucacue.edu.ec \\ Universidad Católica de Cuenca, Azogues \\ Ecuador \\ https://orcid.org/0000-0001-6813-8100 \\ Carlos Marcelo Ávila-Mediavilla \\ cavilam@ucacue.edu.ec \\ Universidad Católica de Cuenca, Cuenca \\ Ecuador \\ https://orcid.org/0000-0002-2649-9634 \\ Juan Carlos Erazo-Álvarez \\ jcerazo@ucacue.edu.ec \\ Universidad Católica de Cuenca, Cuenca \\ Ecuador \\ https://orcid.org/0000-0001-6480-2270
}

Recepción: 10 agosto 2020

Revisado: 25 septiembre 2020

Aprobación: 15 octubre 2020

Publicación: 01 noviembre 2020 


\title{
RESUMEN
}

El objetivo de la investigación consistió en analizar la influencia del nivel instruccional familiar en el proceso de enseñanza aprendizaje en Educación General Básica. Se realizó con coherencia metodológica descriptiva observacional no experimental transversal. En cuanto a las dos variables se coincide que no existe un cumplimiento aceptable en el apoyo de los padres de familia, lo que es un obstáculo para mejorar el rendimiento académico, ya que es un trabajo conjunto con los miembros de la comunidad educativa para conseguir un rendimiento satisfactorio de los estudiantes. Para mejorar la problemática antes mencionada se propone trabajar conjuntamente con la familia en talleres, conformación de grupos e implementar un proyecto que favorezca la disminución del porcentaje de analfabetismo de los representantes de los estudiantes, la calidad de educación depende no solo de los docentes, sino también de los progenitores.

Descriptores: Relación padres-escuela; relación padres-hijos; papel de la familia. (Palabras tomadas del Tesauro UNESCO).

\begin{abstract}
The objective of the research was to analyze the influence of the family instructional level in the teaching-learning process in Basic General Education. It was carried out with descriptive observational non-experimental cross-sectional methodological coherence. Regarding the two variables, it is agreed that there is no acceptable compliance in the support of parents, which is an obstacle to improving academic performance, since it is a joint work with members of the educational community to achieve a satisfactory student performance. To improve the aforementioned problem, it is proposed to work together with the family in workshops, group formation and implement a project that favors the decrease in the percentage of illiteracy of student representatives, the quality of education depends not only on teachers, but also also of the parents.
\end{abstract}

Descriptors: Parent school relationship; parent child relationship; family role. (Words taken from the UNESCO Thesaurus). 


\section{INTRODUCCIÓN:}

En Planificación Administrativa del Ecuador de la zona 6 no se ha dado una investigación de campo planificada y específica sobre este problema, sin embargo, se puede encontrar información dispersa que tiene relación con el caso planteado. De acuerdo al problema de investigación, es imprescindible y necesario conocer la incidencia del impacto mediático del nivel de formación familiar, en todo el transcurso de eficacia y eficiencia en el transcurso de enseñanza-aprendizaje en Básica y la correspondencia de la escuela, comunidad y familia como elementos de la educación, razón por la cual, es importante recalcar que el núcleo de la sociedad es la familia, es quien transmite, educa y forma a sus integrantes para la vida, es decir, promueve el desarrollo integral del futuro ciudadano.

Exclusivamente hablando de la zona rural, se aprecia que el nivel instruccional de los familiares de los estudiantes de Básica, es una problemática grave que se ha detectado mediante la observación en las diferentes aulas y esa percepción se pretende evidenciar con la presente investigación, para que superando las dificultades, ejecutando los lineamientos dados por el Ministerio Nacional de Educación y buscando estrategias pedagógicas, los resultados vayan en beneficio de toda la comunidad educativa en forma general y particularmente de los estudiantes de las instituciones de la zona 6 .

Según estudios realizados por (Mengjie, et al., 2019), el rendimiento académico de los estudiantes se ve afectado por antecedentes familiares como por factores contextuales la diferencia urbano-rural y la variación regional. Se ha evaluado las referencias familiares frente a los factores estructurales para comprobar los logros educativos de los estudiantes en: China, Estados Unidos de América y Alemania, analizando de cinco conjuntos de datos representativos a nivel nacional, de alta calidad y a gran escala. El nivel socioeconómico de la familia ejerce efectos positivos más fuertes sobre el rendimiento académico de los estudiantes en los Estados Unidos y Alemania que en China; $y$ los factores estructurales juegan un papel mucho menor en los Estados Unidos 
y Alemania que en China.

A nivel nacional se registró en el INEC que el analfabetismo es de 6,8\%, en un $20,4 \%$ en los Indígenas, el 7,6\% en los Afroecuatorianos, el 12,9\% en los Montubios, el 5,1\% en los Mestizos, el 3,7\% en los Blancos y el 4,5\% en otro autoidentificación. El analfabetismo en las personas entre 15 o 19 años es del 1,3\% en una tasa menor y el $40,6 \%$ es la tasa mayor en personas de 95 años en adelante con un 40,6\%. Se registra también, que la población de más de 15 años no ha culminado la educación general básica (Décimo año), es decir en un $9 \%$ existe rezago educativo (Instituto Nacional de Estadística y Censos [INEC], 2010).

El Instituto Nacional de Estadística y Censo en la provincia del Cañar registra los siguientes datos relacionados al nivel instruccional de la población, la tasa de asistencia neta es 70,3 en lo que respecta a los comprendidos entre los 15-17 años y el 93,5 con los de 5-14 años. El analfabetismo en la provincia es un 12,2\%. Los niveles de escolaridad predominantes alcanzan un 8,0 los hombres y un 7,3 las mujeres, en un 9,9 lo logran los de las zonas urbanas y un 5,8 los de la zona rural. En cuanto al analfabetismo digital un 32,2 de hombres y 38,6 mujeres (Instituto Nacional de Estadística y Censos [INEC], 2010).

De acuerdo a la información recopilada por la Secretaría Nacional de Planificación y Desarrollo, basada en el último censo realizado en nuestro país, la escolaridad Intercensal de la población en Azogues es de 8,5 años para mujeres y 9,6 años para los hombres. La tasa de analfabetismo es del $10,6 \%$ en las mujeres y el $5,1 \%$ en los hombres, en total es un $8,2 \%$ de analfabetismo en el Cantón (Secretaria Nacional de Planificación y Desarrollo [SENPLADES], 2014).

Esta investigación pretende conocer y analizar la influencia del nivel instruccional de los familiares en el rendimiento académico de los estudiantes de Educación General Básica, para buscar alternativas de solución entre los miembros educativos, involucrando a la familia en la formación de los hijos/as logrando un rendimiento escolar aceptable y la 
misma que vaya en bien del gremio pedagógico reformando ciertos aspectos de la planificación en bien de la educación y en forma específica de los sectores rurales.

El objetivo de la investigación consistió en analizar la influencia del nivel instruccional familiar en el proceso de enseñanza aprendizaje en Educación General Básica.

\section{Referencial teorico}

De acuerdo a datos recopilados por la (Secretaria Nacional de Planificación y Desarrollo, 2015), a nivel nacional el promedio de escolaridad es de 9,6 años de estudio ( 9,5 mujeres y 9,7 hombres), mayor al zonal de 8,9 años (8,5 mujeres y 9,5 hombres). En el área urbana existe mayor promedio de escolaridad tanto en hombres como en mujeres a diferencia de la zona rural. Estableciendo comparación entre los distritos que corresponden a la Zona 6 los que menor nivel de escolaridad tienen son: 01D03, 01D04, 01D05 y 01D08. De acuerdo al sistema educativo actual la tasa neta de asistencia a la educación básica tanto a nivel nacional y zonal es del $93 \%$ habiendo equidad en hombres y mujeres. El distrito 14D05 presenta la tasa más baja de asistencia.

De acuerdo a la asistencia por niveles de educación: en bachillerato la tasa neta a nivel nacional es del $54 \%$, superior a la zonal que es del $51 \%$ y los distritos $14 \mathrm{D} 05,14 \mathrm{D} 06$, 01D05, 14D03 tienen la menor tasa. A nivel nacional asisten a básica superior es 22\% y a nivel zonal es $24 \%$, siendo en mujeres el $25 \%$ y en hombres el $23 \%$. Los distritos con menos tasa de asistencia son: 14D05, 01D07, 14D06, 01D05 y 14D04. En cuanto al analfabetismo el $7 \%$ corresponde a nivel nacional, el $4 \%$ al sector urbano y el $12 \%$ al sector rural, siendo inferior al indicador zonal $8 \%$; existiendo el $10 \%$ en la población femenina y el $5 \%$ en la masculina. Los distritos con la tasa más elevada en analfabetismo son: 01D05, 03D02, 14D05, 01D08, 01D04, 01D03, $01 \mathrm{D} 06$.

Según estudios ejecutados entre familias latinas aculturalizadas y europeo-americanas sobre la influencia de prácticas y valores culturales en el impulso de las competencias, están profundamente vinculados, porque se considera el gran predominio en el logro 
instruccional de los estudiantes. El contexto educativo del hogar es una de las variables que tiene más relación con el aprendizaje, se incluyen la participación de los padres en la escuela, el conocimiento y opinión que tienen de esta y de sus docentes. Es importante considerar que la falta de involucramiento de las familias en la formación y educación de sus representados, es debido a los largos horarios laborales que cumplen porque pertenecen a sectores vulnerables (Pontificia Universidad Católica de Chile, 2006). Para lograr resultados positivos de aprendizaje, los familiares deben garantizar un clima positivo y favorable, en donde fomenten confianza e interés para incentivarles a los estudiantes a la superación y así prepararles para un futuro mejor (Romagnoli \& Cortese, 2015).

La influencia del nivel educativo de los padres/madres o representantes legales de los estudiantes de Educación Básica es de vital importancia dentro del proceso de enseñanza-aprendizaje, considerando sus condiciones sociales, económicas y culturales lo que contribuye a la formación de las personas, por lo que si el nivel educativo de los familiares es alto, habrá mayor responsabilidad en el desempeño de sus hijos e hijas y en caso de ser inferior el nivel instruccional, será menor el compromiso en el ámbito educativo, pero ellos tienen un compromiso en el apoyo incondicional en inculcar actitudes positivas para superar adversidades y alcanzar una formación aceptable. El nivel instruccional y la desigualdad social se afianzan con tres variables: el castigo, el rechazo y la aceptación, las personas que tienen baja autoestima es porque han experimentado rechazo social. En este estudio se ha enfocado el aspecto de escolaridad familiar en la educación de los hijos e hijas (Razeto, 2016).

\section{La familia}

La familia en el transcurso de la historia se ha encargado en la formación del ser humano, por lo que es definida desde varias disciplinas, pero considerando su función que desempeña. De acuerdo con (Mardones Novoa \& Cárcamo Vásquez, 2019) 
conceptualiza a la familia desde el ámbito histórico-social, familia nace con los grupos nómadas, su función económica consistía en que los hombres realizaban actividades de caza y las mujeres sé dedicadas al cuidado de los hijos y luego poco a poco avanzaron en las actividades de la agricultura. Considerando el ámbito jurídico (Villabella, 2016) define a la familia, como el núcleo central de la humanidad dentro de una sociedad ligada por derechos y obligaciones, por lo que la Constitución Nacional debe garantizar leyes que apoyen la permanencia de los miembros de la familia.

Desde la perspectiva psicosocial la familia es el centro evolutivo y de desarrollo de nuestra sociedad, es el pilar fundamental en la preservación humana y en el aporte de valores para la creación de una humanidad justa y equitativa. Por consiguiente, y considerando los elementos mencionados con anterioridad, se concibe la familia como una forma de organización social fundamental para el desarrollo de las personas, entendiendo así que la familia cumple una triple función; económica, afectiva y social, siendo las últimas dos el vínculo fundamental para entender la relación familia-escuela en el contexto educativo (Martínez, 2015).

Según (Razeto, 2016) la familia influye en el desarrollo de los hijos desde la concepción, el cuidado, la atención de los aspectos: económico, social y cultural; también contribuye en el estímulo y desarrollo físico, cognitivo, socioemocional, psicomotriz y del lenguaje. La familia es el modelo que va a seguir para formar una base para su desarrollo personal y académico, de tal manera que se evidenciará en las dimensiones evolutivas y estas características adquiridas, se interconectan con el entorno socializador externo como lo son la escuela y el grupo de iguales.

\section{La escuela}

Actualmente el sistema nacional vigente de Educación del Ecuador tiene tres niveles: Inicial, Básica y Bachillerato. El nivel de Educación Inicial se divide en 2 subniveles: Inicial 1 (3 años), e Inicial 2 (3 a 5 años de edad). El nivel de Educación General Básica se 
divide en 4 subniveles: Preparatoria $1^{\circ}$ grado ( 5 años de edad), Básica Elemental es $2^{\circ}$, $3^{\circ}$ y $4^{\circ}$ grados ( 6 a 8 años de edad), Básica Media de $5^{\circ}, 6^{\circ}$ y $7^{\circ}$ grados ( 9 a 11 años de edad) y Básica Superior, de $8^{\circ}, 9^{\circ}$ y $10^{\circ}$ (12 a 14 años de edad) (Ministerio de Educación del Ecuador [MINEDUC], 2016). De acuerdo con el informe de avance del cumplimiento de la agenda 2030 para el desarrollo sostenible de la Secretaria Técnica Planifica Ecuador (2019), Ecuador tiene el objetivo de promover oportunidades de aprendizaje para todos con una educación inclusiva, equitativa y de calidad (Efstathios, 2020).

La familia y la escuela tiene la misma responsabilidad frente al desarrollo de los estudiantes las normas que rigen en el contexto familiar son diferentes con sus propias reglas, actitudes y destrezas. La escuela tiene como labor facilitar las condiciones para que el estudiante se adecúe y afronte su experiencia escolar con: cualidades, actitudes, destrezas y conocimientos adquiridos, en el seno familiar son los que determinan sus características individuales y sociales. De acuerdo a lo citado, las familias y las instituciones educativas tienen su foco puesto en apoyar el aprendizaje y el desarrollo, para fomentar el aprendizaje integral en los estudiantes.

En la actualidad los representantes solicitan poca información a la escuela sobre el proceso educativo de los estudiantes y no comunican asuntos de la vida familiar y del desarrollo de los niños/as que puedan tener incidencia pedagógica. La escuela por su parte, informa a la familia las normas y reglamentos, bajo rendimiento y conducta inadecuada de los educandos, no así los éxitos escolares. En cuanto al aporte de los encargados en los procesos de toma de decisiones, a nivel administrativo y pedagógico, existe una tendencia hacia un tipo de comunicación unidireccional, que va desde la escuela a la familia, ajustada en la voluntad del sistema directivo y de docentes en definir qué, cuánto y cuándo comunicar.

La educación es la base para preparar una generación de calidad, es la conciencia de crear una atmósfera de enseñanza-aprendizaje para que los estudiantes desarrollen el potencial para controlar la personalidad, inteligencia, moral noble, así como las 
habilidades que necesitan dentro de la sociedad, nación y estado. Para que las metas educativas puedan ser logradas, se necesitan esfuerzos para mejorar la calidad de educación. La influencia de motivación familiar es importante en el proceso de aprendizaje de los estudiantes, puesto que necesitan de afectividad de los seres queridos que lo rodean, siendo ellos la inspiración para elaborar satisfactoriamente sus obligaciones dentro del campo educativo (Al Haq, 2020).

\section{Rendimiento académico}

El rendimiento académico establece un indicador del nivel de aprendizaje alcanzado por los estudiantes, representa la validez en la obtención de los objetivos curriculares para las áreas de estudio y en nuestro medio se expresa mediante un promedio cualitativo y cuantitativo; por ello, es necesario de estudiar los determinantes de los logros obtenidos por cada uno de los estudiantes por parte de las instituciones educativas, este fenómeno en el ámbito educativo se ha convertido en un problema social, que preocupa a los docentes de la institución educativa. La intervención sobre el ambiente familiar constituye un interesante campo de actuación para llevar a cabo experiencias de innovación educativa y de educación compensatoria que permitan un acercamiento a la igualdad de oportunidades en la educación (Lastre Meza, López Salazar, \& Alcázar Berrío, 2018) De acuerdo al (Ministerio de Educación del Ecuador [MINEDUC], 2016) el instructivo para la aplicación de la evaluación estudiantil, tiene las siguientes características específicas como: Reconocer las potencialidades de los estudiantes como individuo y como actor dentro de grupos y equipos de trabajo; retroalimentar la misión estudiantil para obtener mejores resultados de aprendizaje durante el periodo académico; estimular la participación en actividades de aprendizaje tanto de forma cualitativa y cuantitativamente, el beneficio en el progreso integral y de los aprendizajes de los estudiantes. De acuerdo al propósito de la evaluación son de tres tipos: Diagnóstica (inicio de un periodo académico), formativa (proceso de aprendizaje) y sumativa (totalizadora de los logros de 
aprendizaje), la evaluación aplicada para los estudiantes con Necesidades Educativas Especiales es la misma que del resto de estudiantes.

De acuerdo al Art. 193, del Reglamento General a la LOEI: los estudiantes deben lograr aprobar los objetivos de aprendizaje planteados en los programas de las diferentes asignaturas y según el nivel o subnivel del Sistema Nacional de Educación. El rendimiento académico para los subniveles de Educación General Básica y de Bachillerato General se da basada en una escala cualitativa y cuantitativa de calificaciones: 9,00-10,00 Domina los Aprendizajes Requeridos (DAR); 7,00-8,99 Alcanza los Aprendizajes Requeridos; 4,01-6,99 Está Próximo a Alcanzar los Aprendizajes Requeridos y 4,00 No Alcanza los Aprendizajes Requeridos. Cada año escolar está conformado de dos quimestres y se tiene que laborar doscientos días, el quimestre tiene veinte semanas el mismo que está dividid en tres parciales y el examen quimestral, los parciales equivales al $80 \%$ y el examen quimestral el $20 \%$. Las instituciones educativas, registran las notas en el sistema automatizado elaborado por el nivel central de Autoridad Educativa Nacional, las cuales permiten la promoción de grado (Ministerio de Educación del Ecuador [MINEDUC], 2016).

Según el Instructivo para la Aplicación de la Evaluación de los estudiantes, la evaluación de los aprendizajes es un proceso de observación, análisis, valoración y registro a los estudiantes, mediante la aplicación de la retroalimentación pedagógica, el mismo que permite a los docentes mejorar o ratificar la metodología que se está aplicando en el proceso de la enseñanza-aprendizaje. Este proceso es un factor que incide en la formación de los estudiantes ya que permite identificar sus fortalezas y debilidades con un único propósito de mejorar y fortalecer el desempeño académico de los estudiantes. (Arroyo, et al., 2020). 


\section{METODOLOGÍA}

Esta investigación en el campo educativo se realizó con coherencia metodológica descriptiva observacional no experimental transversal, porque se planteó medir el nivel instruccional de los padres de familia y la influencia en el proceso de enseñanzaaprendizaje de los estudiantes de Educación General Básica en único momento.

Se trabajó con muestreo aleatorio simple con la colaboración de docentes de la zona 6 y con muestreo aleatoria estratificada con el apoyo de padres de familia de Básica Elemental. La muestra de estudio fue de 90 padres/madres de familia del nivel elemental, y 60 docentes. Se ejecutó en la escuela de Educación General Básica "4 de noviembre" perteneciente a la Provincia del Cañar, Cantón Azogues, con los padres/madres de familia del nivel elemental y con docentes de Educación General Básica de la zona 6.

La recopilación de datos se utilizó la técnica de encuesta: para padres de familia se utilizó un cuestionario de 14 ítems, y para docentes un cuestionario de 7 ítems, se utilizó la plataforma Microsoft Forms, programa que facilita la elaboración de los cuestionarios y la adquisición del respectivo link que fue compartida por medio de grupos de WhatsApp, tanto de docentes como de representantes de los estudiantes. Se aplicó pruebas estadísticas con el programa SPSS, el cual facilito el proceso de análisis de valores y la adquisición de datos estadísticos fiables, considerando en los docentes el 0,702 y en los padres de familia el 0,751 en el Alfa de Cronbach, lo que reconoció el grado de confirmación del instrumento. Los datos recopilados fueron procesados mediante estadística descriptiva y chi cuadrado de Pearson.

\section{RESULTADOS}

Para esta investigación se recopiló datos en tres días, a través de la plataforma Microsoft Forms, se consideró aspectos importantes sobre la influencia del nivel instruccional familiar en el rendimiento académico de los educandos de Educación Básica, a través de un análisis descriptivo, con la prueba de normalidad kolmogorov-Smirnov con profesores 
Alba Janeth Ortiz-Andrade; Darwin Gabriel García-Herrera; Carlos Marcelo Ávila-Mediavilla; Juan Carlos Erazo-Álvarez

y representantes de los estudiantes, consiguiendo resultados de normalidad paramétrica en todas las variantes.

Tabla 1.

El nivel de instrucción del padre de familia.

\begin{tabular}{cccccc}
\hline & & Frecuencia & Porcentaje & Porcentaje válido & Porcentaje acumulado \\
\hline \multirow{4}{*}{ Válidos } & Ninguno & 6 & 6,7 & 6,7 & 6,7 \\
& Educación Básica & 31 & 34,4 & 34,4 & 41,1 \\
& Bachillerato & 39 & 43,3 & 43,3 & 84,4 \\
& Superior & 14 & 15,6 & 15,6 & 100,0 \\
\cline { 2 - 6 } & Total & 90 & 100,0 & 100,0 & \\
\hline
\end{tabular}

Fuente: Elaboración propia.

Tabla 2.

El nivel de instrucción de la madre de familia.

\begin{tabular}{cccccc}
\hline & & Frecuencia & Porcentaje & Porcentaje válido & Porcentaje acumulado \\
\hline \multirow{4}{*}{ Válidos } & Ninguno & 5 & 5,6 & 5,6 & 5,6 \\
& Algunos años de básica & 19 & 21,1 & 21,1 & 26,7 \\
& Educación Básica & 26 & 28,9 & 28,9 & 55,6 \\
& Bachillerato & 23 & 25,6 & 25,6 & 81,1 \\
& Superior & 17 & 18,9 & 18,9 & 100,0 \\
& Total & 90 & 100,0 & 100,0 & \\
\hline
\end{tabular}

Fuente: Elaboración propia.

Al considerar el nivel de instrucción tanto de los padres y madres de familia se puede determinar que el porcentaje acumulado de los hombres en la escala de Ninguno es de $6,7 \%$ en comparación con el de las mujeres que es de 5,6\%. En lo que respecta al nivel de Educación Básica se puede apreciar que el porcentaje de las mujeres es mayor al de los hombres; en tanto que, en el Bachillerato los porcentajes son cercanos e inversos, es decir, que los hombres tienen un $84,4 \%$, mientras que las mujeres un $81,1 \%$. 
Alba Janeth Ortiz-Andrade; Darwin Gabriel García-Herrera; Carlos Marcelo Ávila-Mediavilla; Juan Carlos Erazo-Álvarez

Si consideramos el nivel superior tanto de hombres y mujeres se acercan en sus porcentajes, pero en una mínima cantidad. Lo que se puede notar claramente es que, en la escala de Algunos años de Básica, solo las mujeres han alcanzado estos niveles de educación, no así en el caso de los hombres que ningún encuestado ha respondido esta variable. Por esta razón, se puede deducir que la educación de los hijos e hijas depende en gran medida de las madres que de los padres.

\section{Tabla 3.}

El nivel de instrucción de la madre de familia es y usted controla y ayuda en la colaboración de las tareas escolares de sus hijos. (Prueba de Chi-cuadrado).

\begin{tabular}{|c|c|c|c|c|c|}
\hline & & \multicolumn{3}{|c|}{$\begin{array}{l}\text { Usted controla y ayuda en la colaboración de } \\
\text { las tareas escolares de sus hijos. }\end{array}$} & \multirow[t]{2}{*}{ Total } \\
\hline & & Nunca & A veces & Siempre & \\
\hline & Ninguno & 2 & 0 & 3 & 5 \\
\hline El nivel de & Algunos años de básica & 1 & 6 & 12 & 19 \\
\hline instrucción de la & Educación Básica & 2 & 14 & 10 & 26 \\
\hline \multirow{4}{*}{ madre de familia es } & Bachillerato & 1 & 5 & 17 & 23 \\
\hline & Superior & 0 & 3 & 14 & 17 \\
\hline & Total & 6 & 28 & 56 & 90 \\
\hline & & Valor & gl & \multicolumn{2}{|c|}{ Sig. asintótica (bilateral) } \\
\hline \multicolumn{2}{|c|}{ Chi-cuadrado de Pearson } & $21,244^{a}$ & 8 & \multicolumn{2}{|c|}{0,007} \\
\hline \multicolumn{2}{|c|}{ Razón de verosimilitudes } & 19,186 & 8 & \multicolumn{2}{|c|}{0,014} \\
\hline \multicolumn{2}{|c|}{ Asociación lineal por lineal } & 5115 & 1 & \multicolumn{2}{|c|}{0,024} \\
\hline \multicolumn{2}{|c|}{$\mathrm{N}$ de casos válidos } & 90 & & & \\
\hline
\end{tabular}

Fuente: Elaboración propia.

En los resultados de la Tabla 3 , se puede observar que la variable sobre el nivel instruccional de las madres de familia dentro del valor de Chi-cuadrado de Pearson, es menor al valor crítico establecido, por lo que la hipótesis sería afirmativa, lo que equivale a H1, determinando que el nivel educativo mínimo que alcanzan es de Educación Básica, razón por la cual dificulta el apoyo en la enseñanza de los hijos, siendo las dos variables compatibles, por cuanto la relación es estrechamente directa, por lo que podemos deducir de acuerdo a los resultados reflejados en las encuestas, que si influye el nivel 
instruccional de las madres en el rendimiento académico de las obligaciones como estudiantes de sus hijos.

Dentro de la escala Nunca, A veces y Siempre se observa que la mayoría de las madres apenas alcanzan a cursar Educación General Básica, puesto que desde la antigüedad el género femenino ha sido excluido de la educación, cumpliendo con obligaciones netamente dentro del hogar.

\section{Tabla 4.}

La situación instruccional de los padres/madres de familia de su escuela, qué posibilidades de ayuda permite en el rendimiento escolar de los estudiantes.

\begin{tabular}{cccccc}
\hline & Frecuencia & Porcentaje & Porcentaje válido & $\begin{array}{c}\text { Porcentaje } \\
\text { acumulado }\end{array}$ \\
\hline \multirow{4}{*}{ Válidos } & Nunca & 5 & 8,3 & 8,3 & 8,3 \\
& A veces & 45 & 75,0 & 75,0 & 83,3 \\
& Siempre & 10 & 16,7 & 16,7 & 100,0 \\
& Total & 60 & 100,0 & 100,0 & \\
\hline
\end{tabular}

Fuente: Elaboración propia.

Según los datos proporcionados por los docentes, se puede observar que, la escala de A veces, tiene un porcentaje de $83,3 \%$, lo que indica que los estudiantes no tienes la ayuda necesaria para realizar sus actividades escolares dentro del hogar y esto acarrea a que el rendimiento sea bajo. En lo que se refiere a la escala de Siempre son muy pocos los padres que cumplen con el apoyo hacia sus representados; mientras que en la escala de Nunca, se puede apreciar un 8,3\%, que es un porcentaje mínimo de padres que no cumplen con sus responsabilidades, tomando en consideración el nivel de instrucción que tienen en su mayoría y la situación económica por la que muchas veces se encuentran alejados de sus hogares. 
Alba Janeth Ortiz-Andrade; Darwin Gabriel García-Herrera; Carlos Marcelo Ávila-Mediavilla; Juan Carlos Erazo-Álvarez

Tabla 5.

Los estudiantes de su institución educativa disponen de todos los útiles escolares.

\begin{tabular}{cccccc}
\hline & & Frecuencia & Porcentaje & Porcentaje válido & $\begin{array}{c}\text { Porcentaje } \\
\text { acumulado }\end{array}$ \\
\hline \multirow{4}{*}{ Válidos } & A veces & 55 & 91,7 & 91,7 & 91,7 \\
& Siempre & 5 & 8,3 & 8,3 & 100,0 \\
& Total & 60 & 100,0 & 100,0 & \\
\hline
\end{tabular}

Fuente: Elaboración propia.

De acuerdo al análisis sobre si los estudiantes cuentan con los útiles escolares para el desempeño en sus clases, los docentes contestan en un promedio máximo, en el rango de $A$ veces el $91,7 \%$ y en el rango de Siempre una mínima cantidad de profesores contestan el $8,3 \%$, por lo que se puede deducir que la mayoría de estudiantes no cuentan con todos los materiales necesarios para un buen desempeño en el rendimiento académico, lo que puede ser el factor económico de los padres que no les permitan obtener todo lo necesario. 
Alba Janeth Ortiz-Andrade; Darwin Gabriel García-Herrera; Carlos Marcelo Ávila-Mediavilla; Juan Carlos Erazo-Álvarez

\section{Tabla 6.}

Situación instruccional de los padres/madres de familia de su escuela y padres/madres de familia de su establecimiento controlan y ayudan en la elaboración de las tareas escolares de sus hijos/as.

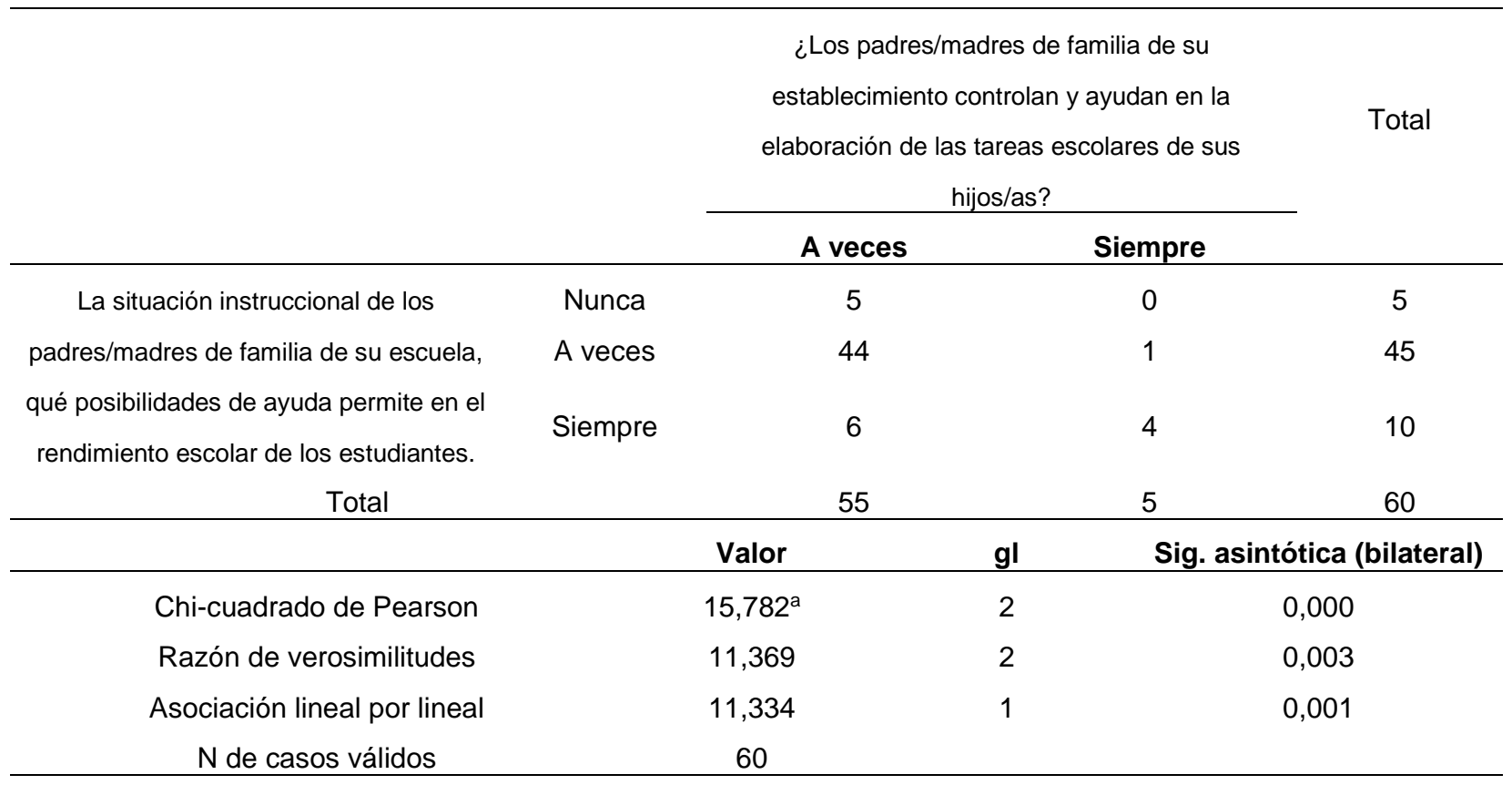

a. 4 casillas $(66,7 \%)$ tienen una frecuencia esperada inferior a 5 . La frecuencia mínima esperada es, 42 .

Fuente: Elaboración propia.

Una vez realizado el análisis de la Tabla 6, sobre la situación instruccional de los padres/madres de familia de los estudiantes de Educación Básica, el valor de Chicuadrado de Pearson, es menor al valor crítico establecido, por lo que la hipótesis sería afirmativa, lo que equivale a $\mathrm{H} 1$, determinando la relación que tiene con la influencia del rendimiento académico de los estudiantes. Las dos variables son compatibles, por cuanto la relación es directamente, lo que permite comprobar debido al bajo nivel instruccional de los representantes es bajo, lo que dificulta el apoyo en el control de tareas de sus hijos. 
Alba Janeth Ortiz-Andrade; Darwin Gabriel García-Herrera; Carlos Marcelo Ávila-Mediavilla; Juan Carlos Erazo-Álvarez

En este sentido la escala de A veces, son apreciadas en un nivel mayor y en Siempre colaboran en las tareas es pequeña, el porcentaje oscila en un $4,00 \%$ y en la escala de A veces el $45,00 \%$ cumple con esta responsabilidad y en el rango de Nunca, existe un mínimo de padres, es decir un 5\% que no se involucra en ningún sentido en el rendimiento académico de sus representados.

En cuanto a las dos variables se coincide que no existe un cumplimiento aceptable en el apoyo de los padres de familia, lo que es un obstáculo para mejorar el rendimiento académico, ya que es un trabajo conjunto con los miembros de la comunidad educativa para conseguir un rendimiento satisfactorio de los estudiantes.

Tabla 7.

Como considera el nivel de rendimiento de su representado.

\begin{tabular}{llcccc}
\hline & Frecuencia & Porcentaje & $\begin{array}{c}\text { Porcentaje } \\
\text { válido }\end{array}$ & $\begin{array}{c}\text { Porcentaje } \\
\text { acumulado }\end{array}$ \\
\hline No alcanza los aprendizajes requeridos. & 1 & 1,1 & 1,1 & 1,1 \\
Está próximo a alcanzar los aprendizajes & 12 & 13,3 & 13,3 & 14,4 \\
requeridos. & 33 & 36,7 & 36,7 & 51,1 \\
Válidos & Alcanza los aprendizajes requeridos. & 34 & 37,8 & 37,8 & 88,9 \\
& Domina los aprendizajes requeridos. & 10 & 11,1 & 11,1 & 100,0 \\
Supera los aprendizajes requeridos. & 90 & 100,0 & 100,0 & \\
\hline
\end{tabular}

Fuente: Elaboración propia.

\section{Tabla 8.}

Rendimiento académico de sus estudiantes.

\begin{tabular}{clcccc}
\hline & Frecuencia & Porcentaje & $\begin{array}{c}\text { Porcentaje } \\
\text { válido }\end{array}$ & $\begin{array}{c}\text { Porcentaje } \\
\text { acumulado }\end{array}$ \\
\hline \multirow{2}{*}{ Válidos } & $\begin{array}{l}\text { Está próximo a alcanzar los } \\
\text { aprendizajes requeridos. }\end{array}$ & 6 & 10,0 & 10,0 & 10,0 \\
$\begin{array}{l}\text { Alcanza los aprendizajes } \\
\text { requeridos. }\end{array}$ & 42 & 70,0 & 70,0 & 80,0 \\
& $\begin{array}{l}\text { Domina los aprendizajes } \\
\text { requeridos. }\end{array}$ & 12 & 20,0 & 20,0 & 100,0 \\
\cline { 2 - 5 } Total & 60 & 100,0 & 100,0 & \\
\hline
\end{tabular}

Fuente: Elaboración propia. 
En cuanto a la relación de la variable del rendimiento de los estudiantes, tanto en la opinión de los representantes de los estudiantes y de los docentes se obtuvo los siguientes resultados: dentro de la escala No alcanza los aprendizajes requeridos tenemos el 1,1\% de los padres de familia, mientras que los docentes no consideran esta variable, en relación a la escala, está próximo a alcanzar los aprendizajes requeridos coinciden tanto docentes como padres de familia teniendo una diferencia mínima, en cambio en la escala.

Alcanzan los aprendizajes requeridos los padres de familia indican que existe un porcentaje del $51,1 \%$, por lo que los docentes contestan en relación a esta pregunta el $80 \%$, notando una clara diferencia en las respuestas de los encuestados, lo que no sucede con la variable de Domina los aprendizajes requeridos, donde existe una notoria diferencia ya que los padres de familia contestan un $88,9 \%$ y los docentes el $20 \%$, finalmente en la variable de Supera los aprendizajes requeridos el $11,1 \%$ mencionan los padres de familia, mientras que los docentes no indican sobre este rango, por lo que podemos deducir, en lo que hace referencia al rendimiento de los estudiantes los padres de familia indican que sus hijos dominan los aprendizajes requeridos, situación que no concuerda con los profesores, por cuanto ellos indican que solo un mínimo porcentaje de estudiantes cumplen con este rango.

En torno a estudios realizados por (Murillo \& Castilla, 2020) en escuelas latinoamericanas para explorar el efecto de las diferentes formas de participación de los padres en el rendimiento de los estudiantes de primaria. La participación de los padres en el proceso educativo y en la escuela influye directamente en el rendimiento académico del estudiante. Los resultados muestran que el rendimiento escolar, se ve influido por sus niveles socio-económicos y educativos, idioma nativo, género y del entorno de vida (rural/urbano). 
En los países latinoamericanos registran que reciben ayuda en sus tareas por parte de la madre en un 90\% estudiantes de Cuba, Panamá, Chile y República Dominicana, y más del $80 \%$ en los países latinoamericanos restantes, excepto para Guatemala y Uruguay. La cantidad de apoyo paterno, por el contrario, varió del 48\% (Uruguay) al 80\% (Cuba), lo que indica que los padres, aunque son una fuente importante de ayuda, constituyen un recurso utilizado con menos frecuencia que las madres. La familia es primordial en el aspecto educativo, en la formación personal, en el contexto dentro de la sociedad, se puede concluir que la familia tiene una relación con el rendimiento escolar y la formación integral de los estudiantes en todos los niveles de instrucción.

\section{PROPUESTA}

Según la investigación realizada sobre el nivel instruccional de los padres/madres de familia y su influencia en el rendimiento académico de los estudiantes de Educación General Básica, se ha detectado, que este, es un problema real grande, razón por la cual se sugiere alternativas de solución, mismas que se presenta a continuación:

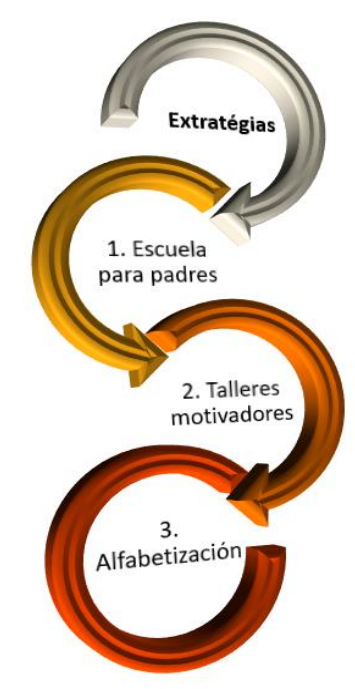

Figura 1. Estrategias para mejorar el nivel instruccional familiar y su influencia en el rendimiento académico de los estudiantes.

Fuente: Elaboración propia. 
1. Escuela para padres: Es considerada como una de las alternativas para solucionar en parte nuestra problemática ya que, son asambleas de trabajo con información, formación y reflexión sobre las tareas y responsabilidades que deben cumplir dentro de la formación educativa los representantes de los estudiantes, misma que está conformada de varios grupos, responsabilizados los miembros del Departamento de Consejería Estudiantil (DECE) o los tutores de cada grado, quienes se encargan de seleccionar los temas a trabajar y preparar materiales necesarios para dirigir las charlas, también se puede buscar apoyo de otras organizaciones para indagar alternativas de solución a las dificultades detectadas.

Esta aplicación de Escuela para Padres tiene como objetivo facilitar información, orientación, formación y asesoramiento para su importante tarea de actuar como tales y, lograr así, un buen desarrollo de la personalidad y en rendimiento académico aceptable de sus hijos/hijas. Este programa está destinado a fortalecer el núcleo más importante de la sociedad que es la familia, dando una oportunidad para compartir vivencias, analizar y reflexionar y así, conjuntamente buscar alternativas de solución a los problemas asociados con el rendimiento escolar.

Estos proyectos dentro de las instituciones educativas permiten brindar una educación para el amor y la vida, para contribuir en la educación, el crecimiento y desarrollo personal, familiar y social, para mejorar las condiciones de vida de las familias generando propuestas de educación que contribuyan al bienestar familiar compartiendo vivencias y practicando valores, aceptando las responsabilidades y obligaciones en el proceso de formación de los hijos, permitiendo reafirmar, la unidad familiar básica de la sociedad y participación libre, justa y democrática.

2. Talleres motivadores para padres y familiares: Los talleres son espacios educativos alternativos que permiten promover procesos de conocimientos y de vinculación social, facilitando la solución de problemas, se compone de un lugar de 
Alba Janeth Ortiz-Andrade; Darwin Gabriel García-Herrera; Carlos Marcelo Ávila-Mediavilla; Juan Carlos Erazo-Álvarez

co-aprendizaje, donde los participantes construyen socialmente conocimientos y valores, desarrollan habilidades y actitudes, a partir de sus adecuadas experiencias dentro de este espacio, sin embargo, se diferencian los roles de aprendiz y de facilitador, en función de la mejoría de la calidad del producto colectivo de trabajo, para los cuales se puede tratar sobre las siguientes temáticas: Familia y educación, conociendo a nuestros hijos, educar en la libertad, cómo comunicarnos en familia, la crítica negativa, formación en valores humanos, formación de hijos para la no violencia, tiempo compartido en familia, quién es la autoridad dentro del núcleo familiar, nuestros hijos y la toma de decisiones en la elección profesional. Para lograr con los objetivos de los talleres se debe involucrar a:

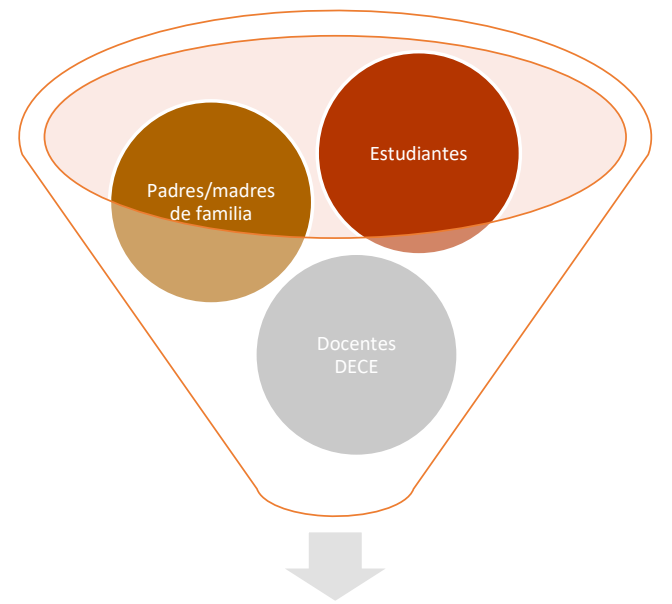

\section{Involucrados}

Figura 2. Involucrados para los talleres motivacionales.

Fuente: Elaboración propia. 
Alba Janeth Ortiz-Andrade; Darwin Gabriel García-Herrera; Carlos Marcelo Ávila-Mediavilla; Juan Carlos Erazo-Álvarez

Recomendaciones para realizar los talleres con padres/madres de familia, para que puedan aprender y crecer juntos durante las sesiones, es muy importante que se cumplan algunas reglas:

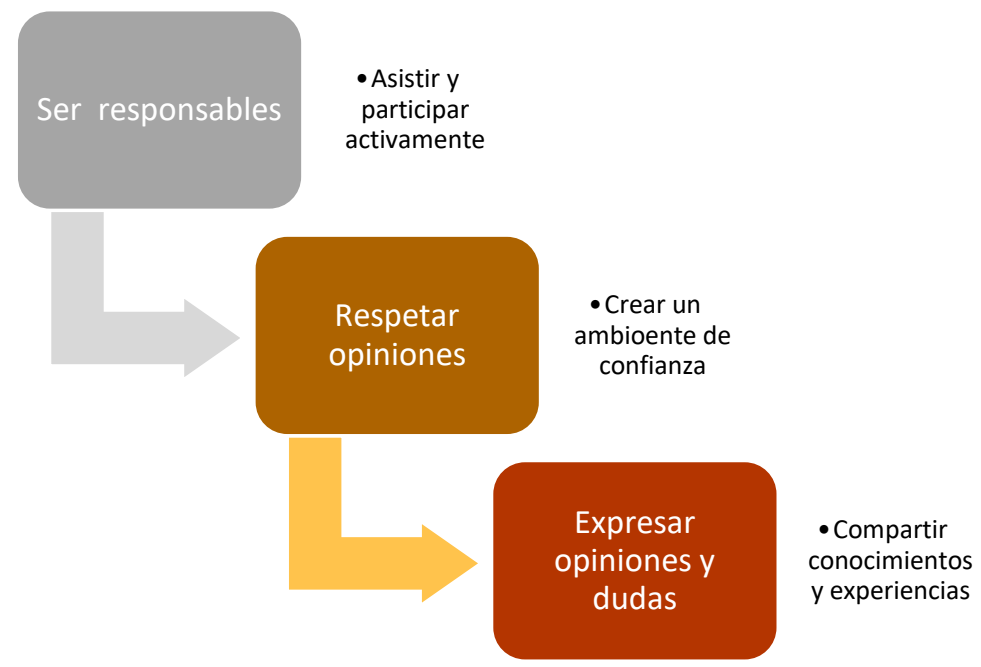

Figura 3. Reglas para asistir a talleres motivacionales.

Fuente: Elaboración propia.

3. Alfabetización a padres/madres se sectores rurales: $\mathrm{El}$ analfabetismo es considerado la incapacidad de leer y escribir que se debe habitualmente a la falta de enseñanza de las mismas capacidades, las cuales se originan con la mayoría de personas de sectores rurales, por lo que el Ministerio de Educación del Ecuador, dentro de la Subsecretaria de Coordinación Educativa, implementó el proyecto de Alfabetización a personas adultas, pero no se han cubiertos los lugares alejados de las zonas rurales, para lo cual se debe implementar un proyecto de alfabetización dentro de las instituciones educativas para poder contribuir con los representantes a que aprender a leer y a escribir, ya que es uno de los factores que más afecta en la formación de los hijos e hijas por parte de los progenitores, pues algunos no cuentan con ningún nivel educativo, lo que no les permite cumplir responsablemente el papel 
Alba Janeth Ortiz-Andrade; Darwin Gabriel García-Herrera; Carlos Marcelo Ávila-Mediavilla; Juan Carlos Erazo-Álvarez

en la formación educativa de sus representados, este proyecto permitirá reducir la tasa de analfabetismo en nuestro entorno. Implementar el proyecto denominado Familia Apoyo y Superación para Aprender (FASA), con el que se pretende involucrar y comprometer a los integrantes de la familia, en cada etapa se ejecutará un proceso para lograr la meta de alfabetización a los representantes que lo requiera, el proyecto consistirá en:

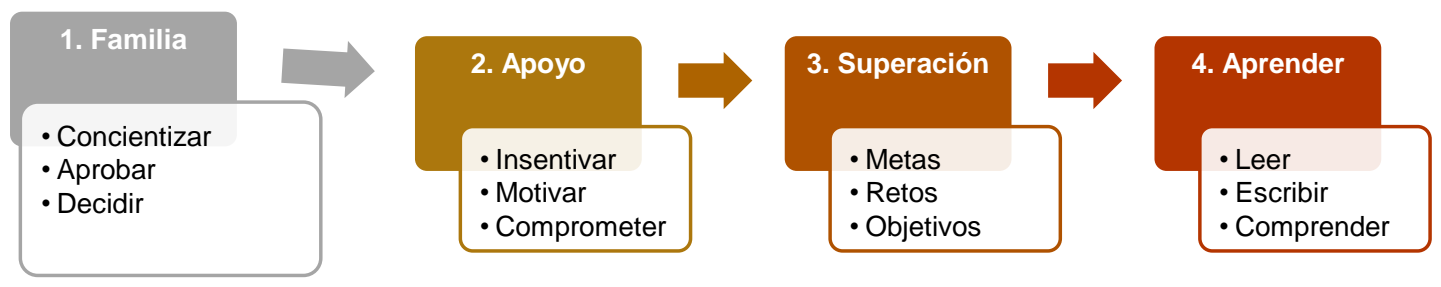

Figura 4. Esquema de implementación de proyecto de alfabetización.

Fuente: Elaboración propia.

Por todo lo antes expuesto se debe recalcar que los padres que tienen un bajo nivel instruccional, deben dejar ayudarse por las instituciones que están encargados de mejorar la brecha del analfabetismo, contribuyendo de esa manera a mejorar el rendimiento académico de sus hijos e hijas en las diferentes instituciones educativas.

\section{CONCLUSIONES}

El nivel instruccional de los progenitores o representantes si influyen en el rendimiento académico de los estudiantes de Educación General Básica, debido a que la mayoría $(26,7 \%)$ de familiares no tienen estudios básicos que orienten al buen desempeño académico de los hijos.

Según análisis emitidos por parte de los docentes y padres/madres de familia son un mínimo de estudiantes que superan los aprendizajes requeridos, por lo que se ha comprobado que la mayoría de estudiantes que provienen de sectores rurales no pueden 
tener un buen desempeño en la educación debido a factores socio-económicos y educativos.

Para mejorar la problemática antes mencionada se propone trabajar conjuntamente con la familia en talleres, conformación de grupos e implementar un proyecto que favorezca la disminución del porcentaje de analfabetismo de los representantes de los estudiantes, para que de esta manera los involucrados puedan desempeñar favorablemente sus responsabilidades dentro del espacio educativo, ya que la calidad de educación depende no solo de los docentes, sino también de los progenitores, propuesta abordada en el presente artículo.

La característica fundamental de este tema fue buscar y proponer alternativas de solución para poder mejorar el rendimiento académico de los estudiantes en todos los niveles de Educación Básica, para poder trabajar conjuntamente con los representantes construir nuevas expectativas en la educación de los miembros de los sectores rurales.

\section{FINANCIAMIENTO}

No monetario

\section{AGRADECIMIENTOS}

A la Escuela de Educación General Básica "4 de noviembre" perteneciente a la Provincia del Cañar, Cantón Azogues; por apoyar el desarrollo de la investigación.

\section{REFERENCIAS}

Al Haq, V. (2020). Effect of Learning Motivation and Learning Environment Against Student Learning Achievement. [Efecto de la motivación del aprendizaje y el entorno de aprendizaje contra el logro de aprendizaje de los estudiantes]. Early Childhood Research Journal, 2(1); 6-11. 
Alba Janeth Ortiz-Andrade; Darwin Gabriel García-Herrera; Carlos Marcelo Ávila-Mediavilla; Juan Carlos Erazo-Álvarez

Arroyo, E., Solórzano, M., Zambrano, M., \& Luque, K. (2020). Feedback and Improvement on Teaching-learning Process for Basic General Education Students. [Retroalimentación y mejora del proceso de enseñanza-aprendizaje para estudiantes de educación general básica]. International Journal of Social Sciences, 3(1), 39-46.

Efstathios, S. (2020). ¿Quiénes son los Pobres Ecuatorianos por Ingresos? Una mirada a través de la educación. [Who are the Poor Ecuadorians by Income? A look through education]. Cuenca-Ecuador: Centro de Estudio Sociales de América Latina (CES-AL.). Recuperado de https://n9.cl/uqc5h

Instituto Nacional de Estadística y Censos [INEC]. (2010). El Censo informa: Educación. [The Census reports: Education]. Recuperado de https://n9.cl/i7zor

Instituto Nacional de Estadística y Censos [INEC]. (2010). Fascículo provincial Cañar INEC. [Provincial Fascicle Cañar INEC]. Recuperado de https://n9.cl/8r9j4

Lastre Meza, K., López Salazar, L. D., \& Alcazar Berrio, C. (2018). Relación entre apoyo familiar y el rendimiento académico en estudiantes colombianos de educación primaria. [Relationship between family support and academic achievement in primary school students]. Psicogente, 21(39), 102-115. https://doi.org/10.17081/psico.21.39.2825

Mardones Novoa, R., \& Cárcamo Vásquez, H. (2019). Familia y escuela en el marco de la Ley 20.911 de formación ciudadana. [Family and school within the framework of Law 20,911 on citizen training]. UCMaule, (57), 99-114. https://doi.org/10.29035/ucmaule.57.99

Martínez, H. (2015). La familia: una visión interdisciplinaria. [The family: an interdisciplinary vision]. Revista Médica Electrónica, 37(5), 523-534.

Mengjie, L., Wangyang, L., \& Yu, X. (2019). The influences of family background and structural factors on children's academic performances: A cross-country comparative study. [Las influencias de los antecedentes familiares y los factores estructurales en el rendimiento académico de los niños]. Chinese Journal of Sociology, 5(2) 173-192 
Alba Janeth Ortiz-Andrade; Darwin Gabriel García-Herrera; Carlos Marcelo Ávila-Mediavilla; Juan Carlos Erazo-Álvarez

Ministerio de Educación del Ecuador [MINEDUC]. (2016). Currículo de los niveles de educación obligatoria. [Curriculum of compulsory education levels]. Quito: Ministerio de Educación del Ecuador. Recuperado de https://n9.cl/mnlj

Ministerio de Educación del Ecuador [MINEDUC]. (2016). Instructivo para la aplicación de la evaluación estudiantil. [Instructions for the application of the student assessment]. Quito: Ministerio de Educación. Recuperado de https://n9.cl/et9u

Murillo, F., \& Castilla, R. (2020). ¿La implicación de las familias influye en el rendimiento? Un estudioen educación primaria en América Latina. [Does family involvement influence performance? A study in primary education in Latin America]. Revista de Psicodidáctica, 25(1); 13-22.

Pontificia Universidad Católica de Chile. (2006). Familia y proceso de aprendizaje. [Family and learning process]. Villarrica-Chile: Gobierno de Chile. Recuperado de https://n9.cl/8jgp

Razeto, A. (2016). El involucramiento de las familias en la educación de los niños: Cuatro reflexiones para fortalecer la relación entre familias y escuelas. [The parent involvement in the education of the children. Four reflections to strengthen the relation between families and schools]. Páginas de Educación, 9(2), 184-201.

Razeto, A. (2016). El involucramiento de las familias en la educación de los niños. [The involvement of families in the education of children]. Revista Páginas de Educación, 9(12)

Romagnoli, C., \& Cortese, I. (2015). ¿Cómo la familia influye en el aprendizaje y rendimiento escolar? [How does the family influence learning and school performance?]. Valoras UC, 1-6. Obtenido de https://n9.cl/yue0

Secretaria Nacional de Planificación y Desarrollo [SENPLADES]. (2014). Ficha de cifras generales SEMPLADES. [SEMPLADES general figures sheet]. Recuperado de https://n9.cl/4pp7w

Secretaria Nacional de Planificación y Desarrollo. (2015). Agenda Zonal Provincias de Azuay, Cañar y Morona Santiago [Zonal Agenda Provinces of Azuay, Cañar and Morona Santiago]. Quito-Ecuador: Senplades/1a edición. Recuperado de https://n9.cl/xlzy 
EPISTEME KOINONIA

Revista Electrónica de Ciencias de la Educación, Humanidades, Artes y Bellas Artes

Año III. Vol III. N¹. Edición Especial. 2020

Hecho el depósito de Ley: FA2018000022

ISSN: 2665-0282

FUNDACIÓN KOINONIA (F.K).

Santa Ana de Coro, Venezuela

Alba Janeth Ortiz-Andrade; Darwin Gabriel García-Herrera; Carlos Marcelo Ávila-Mediavilla; Juan Carlos Erazo-Álvarez

Villabella, C. (2016). Constitución y familia. Un estado comparativo [Constitution and family. A comparative state]. Díkaion, 25(1),100-131.

(C2020 por el autor. Este artículo es de acceso abierto y distribuido según los términos y condiciones de la licencia CreativeCommons Atribución-NoComercial-Compartirlgual 4.0 Internacional (CC BY-NC-SA 4.0) (https://creativecommons.org/licenses/by-nc-sa/4.0/). 